\title{
Cationic Covalent Organic Frameworks for Fabricating Efficient Triboelectric Nanogenerator
}

Lipeng Zhai, ${ }^{\dagger}$ Wutao Wei, ${ }^{\dagger}$ Baiwei Ma,${ }^{\dagger}$ Wanyu Ye,${ }^{\dagger}$ Jing Wang, ${ }^{\dagger}$ Weihua Chen,,${ }^{\ddagger}$ Xiubei Yang,,${ }^{\dagger}$ Siwen Cui, ${ }^{\dagger}$ Zijie Wu, ${ }^{\|}$Constantinos Soutis, ${ }^{\|}$Guangshan Zhu, ${ }^{{ }^{*}}$ Liwei $\mathrm{Mi}^{+*}$

$\dagger$ : Center for Advanced Materials Research, Henan Key Laboratory of Functional Salt Materials, Zhongyuan University of Technology, Zhengzhou 450007 (P. R. China)

†: College of Chemistry, Zhengzhou University Zhengzhou 450001 (P. R. China)

I: North West Composites Center, School of Materials, University of Manchester, Manchester M13 9PL (UK)

$\S:$ Key Lab of Polyoxometalate Science of Ministry of Education Faculty of Chemistry, Northeast Normal University Changchun 130024 (P. R. China) 


\section{Contents}

Section A. Materials and Methods

Section B. Synthetic Procedures

Section C. Supporting Figures

Section D. Supporting Tables

Section E. Supporting References 


\section{Section A. Materials and Methods}

${ }^{1} \mathrm{H}-\mathrm{NMR}$ spectra were measured on JEOL models JNM-LA400 NMR spectrometer, where chemical shifts ( $\delta$ in $\mathrm{ppm})$ were determined with a residual proton of the solvent as standard. Fourier transform infrared (FT IR) spectra were recorded on a JASCO model FT IR-6100 infrared spectrometer. X-ray diffraction (XRD) data were recorded on a Rigaku model RINT Ultima III diffractometer by depositing powder on glass substrate, from $2 \theta=1.5^{\circ}$ up to $30^{\circ}$ with $0.02^{\circ}$ increment. Elemental analysis was performed on a Yanako CHN CORDER MT-6 elemental analyzer. TGA measurements were performed on a Mettler-Toledo model TGA/SDTA851e under $\mathrm{N}_{2}$, by heating to $800{ }^{\circ} \mathrm{C}$ at a rate of $10^{\circ} \mathrm{C} \mathrm{min}^{-1}$.

Nitrogen sorption isotherms were measured at $77 \mathrm{~K}$ with a Micromeritics Instrument Corporation model 3Flex surface characterization analyzer. Before measurement, the samples were degassed in vacuum at $120{ }^{\circ} \mathrm{C}$ for more than $10 \mathrm{~h}$. The Brunauer-Emmett-Teller (BET) method was utilized to calculate the specific surface areas. By using the non-local density functional theory (NLDFT) model, the pore volume was derived from the sorption curve. Four-probe electrode was conducted on the Beijing Meihua Instrument Technology Co LTD (MHY-7756).

The crystalline structures of COFs were determined using the density-functional tight-binding $(\mathrm{DFTB}+)$ method including Lennard-Jones (LJ) dispersion. The calculations were carried out with the DFTB+ program package version 1.2. DFTB is an approximate density functional theory method based on the tight binding approach and utilizes an optimized minimal LCAO Slater-type all-valence basis set in combination with a two-center approximation for Hamiltonian matrix elements. The Coulombic interaction between partial atomic charges was determined using the self-consistent charge (SCC) formalism. Lennard-Jones type dispersion was employed in all calculations to describe van der Waals (vdW) and $\pi$-stacking interactions with starting structures created by AuToGraFSS1 and pre-optimized using a topology-preserving force field were used to optimize the monolayer and were further extended to layered frameworks with different stacking modes. The lattice dimensions were optimized simultaneously with the geometry. Standard DFTB parameters for 
$\mathrm{X}-\mathrm{Y}$ element pair $(\mathrm{X}, \mathrm{Y}=\mathrm{C}, \mathrm{H}, \mathrm{N}$, and $\mathrm{Br}$ ) interactions were employed from the mio-0-1 set10. The accessible surface areas were calculated from the Monte Carlo integration technique using a nitrogen-size probe molecule (diameter $=3.68 \AA$ ) roll over the framework surface with a grid interval of $0.25 \AA$. The XRD pattern simulation was performed in a software package for crystal determination from PXRD pattern, implemented in MS modeling version 4.4 (Accelrys Inc.). We performed Pawley refinement to optimize the lattice parameters iteratively until the $R_{\mathrm{P}}$ and $R_{\mathrm{WP}}$ values converge. The pseudo-Voigt profile function was used for whole profile fitting and Berrar-Baldinozzi function was used for asymmetry correction during the refinement processes. The final $R_{\mathrm{WP}}$ and $R_{\mathrm{P}}$ values were $5.44 \%$ and $4.21 \%$ for TFP-DB-COF, respectively. The final $R_{\mathrm{WP}}$ and $R_{\mathrm{P}}$ values were $7.96 \%$ and $5.74 \%$ for TFP-DP-COF, respectively.

Computational method: We have employed the Vienna Ab initio Simulation Package ${ }^{\text {S1-2 }}$ to perform all density functional theory (DFT) calculations within the generalized gradient approximation (GGA) using the Perdew-Burke-Ernzerhof (PBE) ${ }^{\mathrm{S} 3}$ formulation. We have chosen the projected augmented wave (PAW) potentials ${ }^{\mathrm{S} 4-5}$ to describe the ionic cores and take valence electrons into account using a plane wave basis set with a kinetic energy cutoff of $450 \mathrm{eV}$. Partial occupancies of the Kohn-Sham orbitals were allowed using the Gaussian smearing method and a width of $0.05 \mathrm{eV}$. The electronic energy was considered self-consistent when the energy change was smaller than $10^{-5} \mathrm{eV}$. A geometry optimization was considered convergent when the energy change was smaller than $0.03 \mathrm{eV}^{*} \AA^{-1}$. During structural optimizations of the COF models, a $1 \times 2 \times 1 \mathrm{k}$-point grid for Brillouin zone was used.

\section{COFs-based TENG output performance measurements}

The short circuit current $\left(I_{\mathrm{sc}}\right)$ and the output voltage $\left(V_{\mathrm{o}}\right)$ were tested by a SR570 low-noise current amplifier (Stanford Research System) and a NI-PCI6259 (National Instruments) with a load resistor of $100 \mathrm{M} \Omega$, respectively. The test temperature and humidity are $23 \pm 2{ }^{\circ} \mathrm{C}$ and $35 \pm 1 \%$, respectively.

Resistance measurement. Impedance analyses were performed on COF powders. The powders were obtained by grinding the samples. The resultant powders (200 mg 
for each COF) were added into a 10-mm standard die and then slowly increased pressure to $10 \mathrm{kN}$ and kept for $5 \mathrm{~min}$ to prepare pellets. Measurements were performed using an impedance analyzer (CHI660D electrochemical workstation), with a two-Ag-probe over the frequency range from $4 \mathrm{~Hz}$ to $5 \mathrm{MHz}$ and with an input voltage amplitude of $100 \mathrm{mV}$. The cell was filled with nitrogen before conducting the measurements. The conductivity $(\sigma)$ was obtained from equation: $\sigma=\mathrm{L} /(\mathrm{Z} \times \mathrm{S})$, where the thickness of sample was showed by $\mathrm{L}(\mathrm{cm})$, electrode area was represented by $\mathrm{S}\left(\mathrm{cm}^{2}\right)$ and, impedance was indicated by $\mathrm{Z}(\Omega)$. 


\section{Section B. Synthetic Procedures}

Mesitylene, 1, 4-dioxane, tetrahydrofuran, dimethylacetamide, acetic acid, dimidium bromide (DB), and 3,8-diamino-6-phenylphenanthridine (DP) were purchased from Aladdin chemicals and used without further purification unless otherwise noted. V mode $0.1 \mathrm{~F}$ Capacitor with $5.5 \mathrm{~V}$ was purchased from TaoBao website. 1,3,5-triformylphloroglucinol (TFP) were synthesized according to the reported method. ${ }^{[\mathrm{S} 6]}$

Synthesis of TFP-DB-COF: A pyrex tube $\left(\begin{array}{lll}10 & \mathrm{ml}\end{array}\right)$ is charged with 1,3,5-triformylphloroglucinol (TFP) $(0.1 \mathrm{mmol})$, dimidium bromide (DB) $(0.15$ mmol), $1 \mathrm{~mL}$ 1,4-dioxane/mesitylene (v/v, 1:1), $0.1 \mathrm{~mL}$ of $6 \mathrm{M}$ aqueous acetic acid. The tube was then flash frozen at $77 \mathrm{~K}$ and degassed by three freeze-pump-thaw cycles. The tube was sealed off and then heated at $120{ }^{\circ} \mathrm{C}$ for 3 days. The powder collected was washed with dimethylacetamide, tetrahydrofuran and methanol and dried at $100{ }^{\circ} \mathrm{C}$ under vacuum for 12 hours to get corresponding red powder in $\sim 76 \%$ isolated yield.

Synthesis of TFP-DP-COF: A pyrex tube $(10 \mathrm{ml})$ is charged with TFP $(0.1 \mathrm{mmol})$, 3,8-diamino-6-phenylphenanthridine (DP) (0.15 mmol), $1 \mathrm{~mL} \mathrm{1,4-dioxane/mesitylene}$ $(\mathrm{v} / \mathrm{v}, 1: 1), 0.1 \mathrm{~mL}$ of $6 \mathrm{M}$ aqueous acetic acid. The tube was then flash frozen at $77 \mathrm{~K}$ and degassed by three freeze-pump-thaw cycles. The tube was sealed off and then heated at $120{ }^{\circ} \mathrm{C}$ for 3 days. The collected powder was washed with dimethylacetamide, tetrahydrofuran and methanol and dried at $100{ }^{\circ} \mathrm{C}$ under vacuum for 12 hours to get corresponding red powder in $\sim 77 \%$ isolated yield.

Fabrication of COFs-based TENG: The negative triboelectric electrode with PVDF was prepared according to the literature. ${ }^{[\mathrm{S} 7]}$ Unlike the preparation process of PVDF electrode, COF samples (5 mg) were preground mechanically, and then paved uniformly and fixed firmly on the sticky side of the $\mathrm{Cu}$ tape. An air gun was used to remove the redundant powder. Finally, a $\mathrm{Cu}$ wire was attached on the other side of the $\mathrm{Cu}$ tape by conductive silver epoxy. Combine the positive part with COF materials and the negative part with PVDF together, the COFs-based TENG was obtained. The 
vertical contact-separation mode of TENG was used, and the effective contact area is $5 \times 5 \mathrm{~cm}^{2}$. 
Section C. Supporting Figures

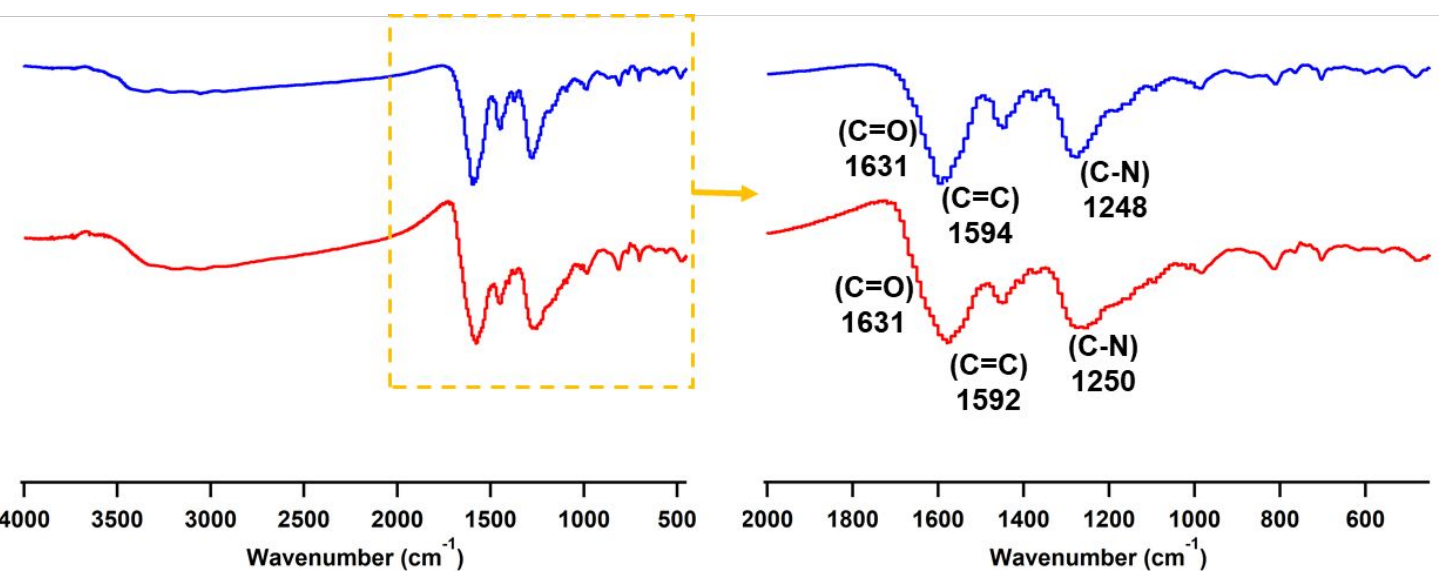

Figure S1. FT IR spectra of TFP-DP-COF (blue curve) and TFP-DB-COF (red curve). 


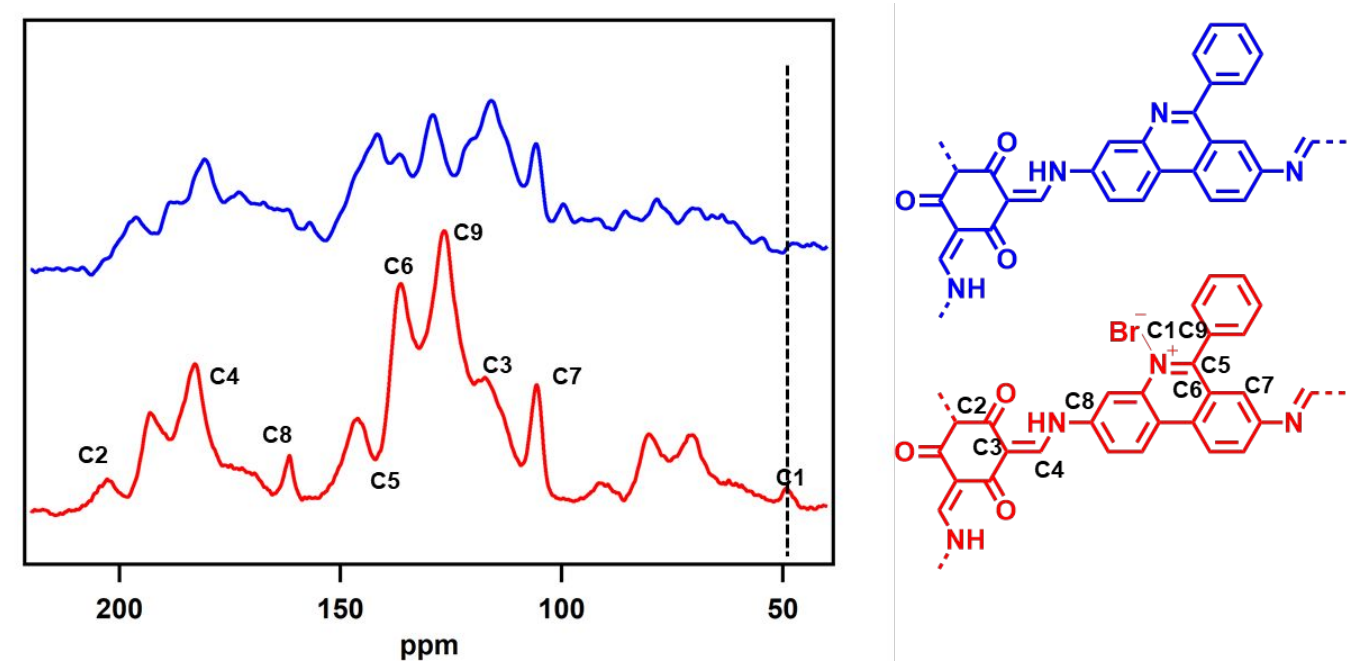

Figure S2. ${ }^{13} \mathrm{C}$ CP-MAS solid-state NMR spectra of TFP-DP-COF (blue curve) and TFP-DB-COF (red curve). 


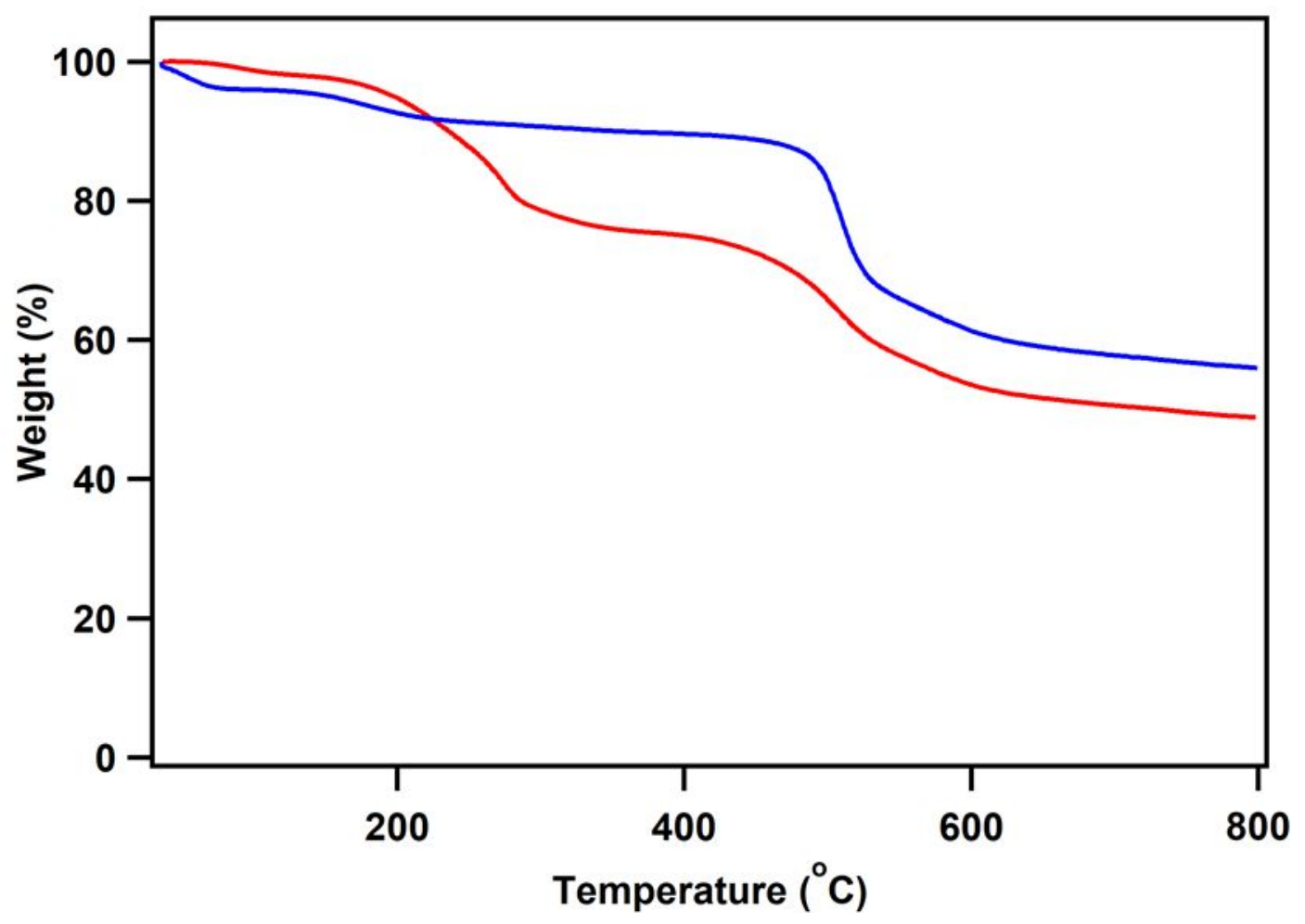

Figure S3. TGA curves of TFP-DP-COF (blue curve) and TFP-DB-COF (red curve). 


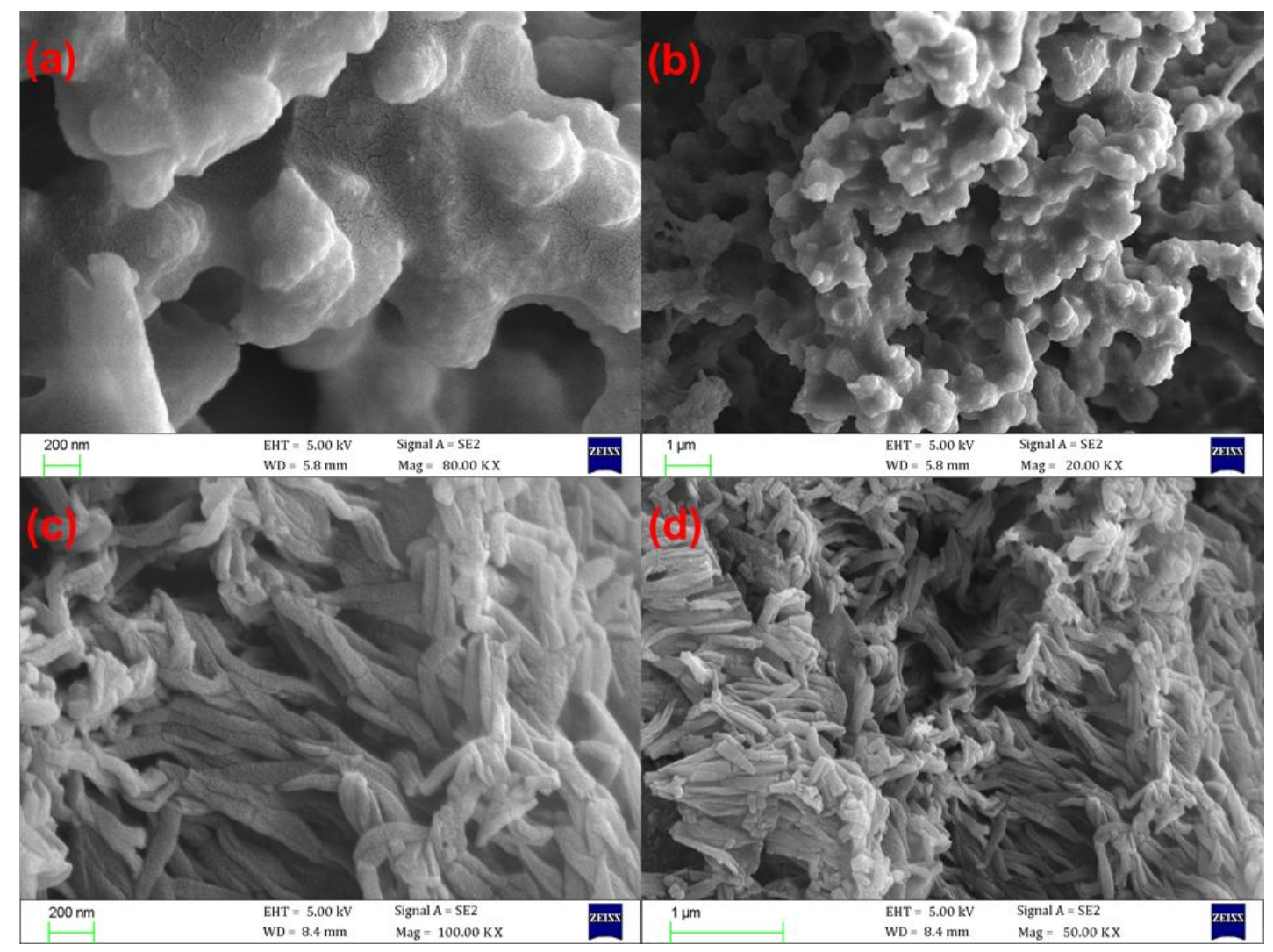

Figure S4. SEM images of TFP-DP-COF $(a, b)$ and TFP-DB-COF (c, d). 


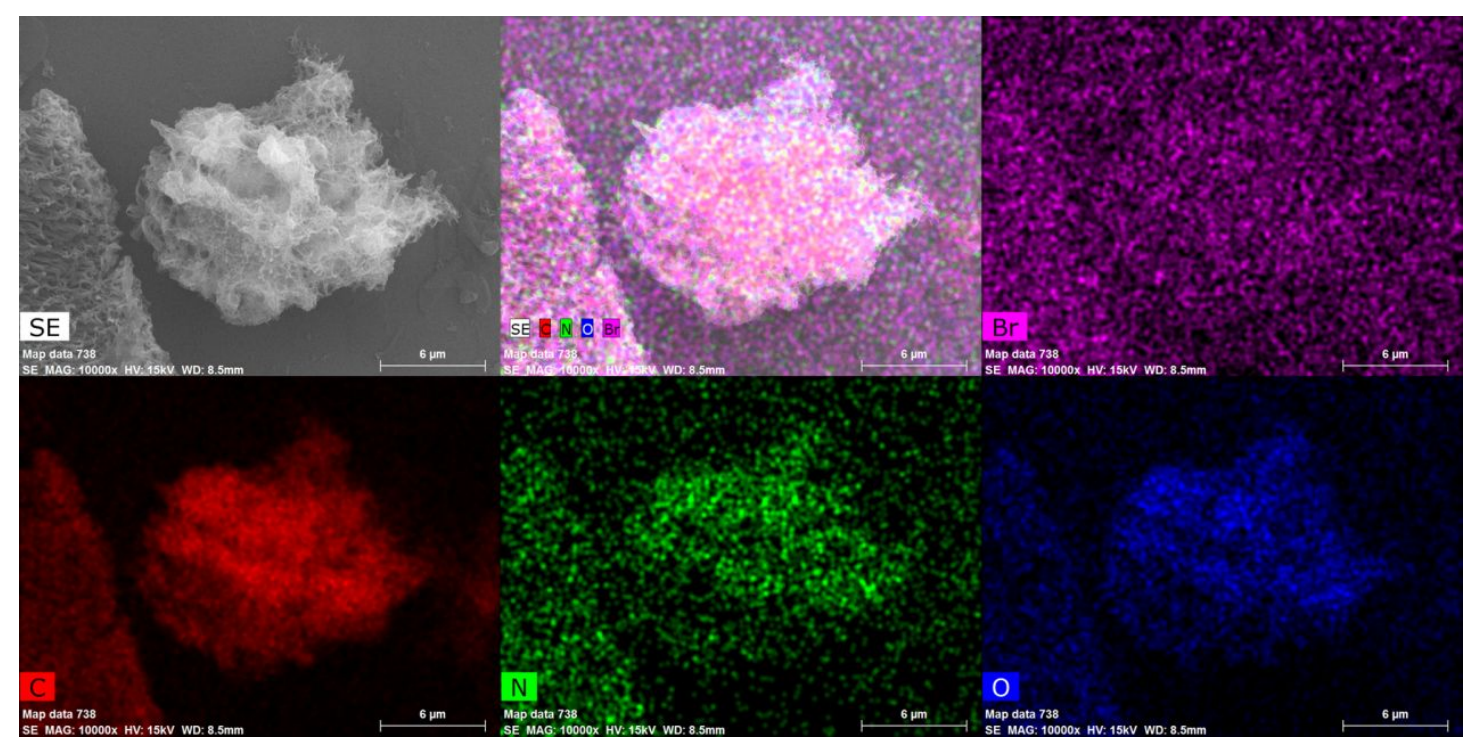

Figure S5. The EDX elemental mapping of TFP-DB-COF.

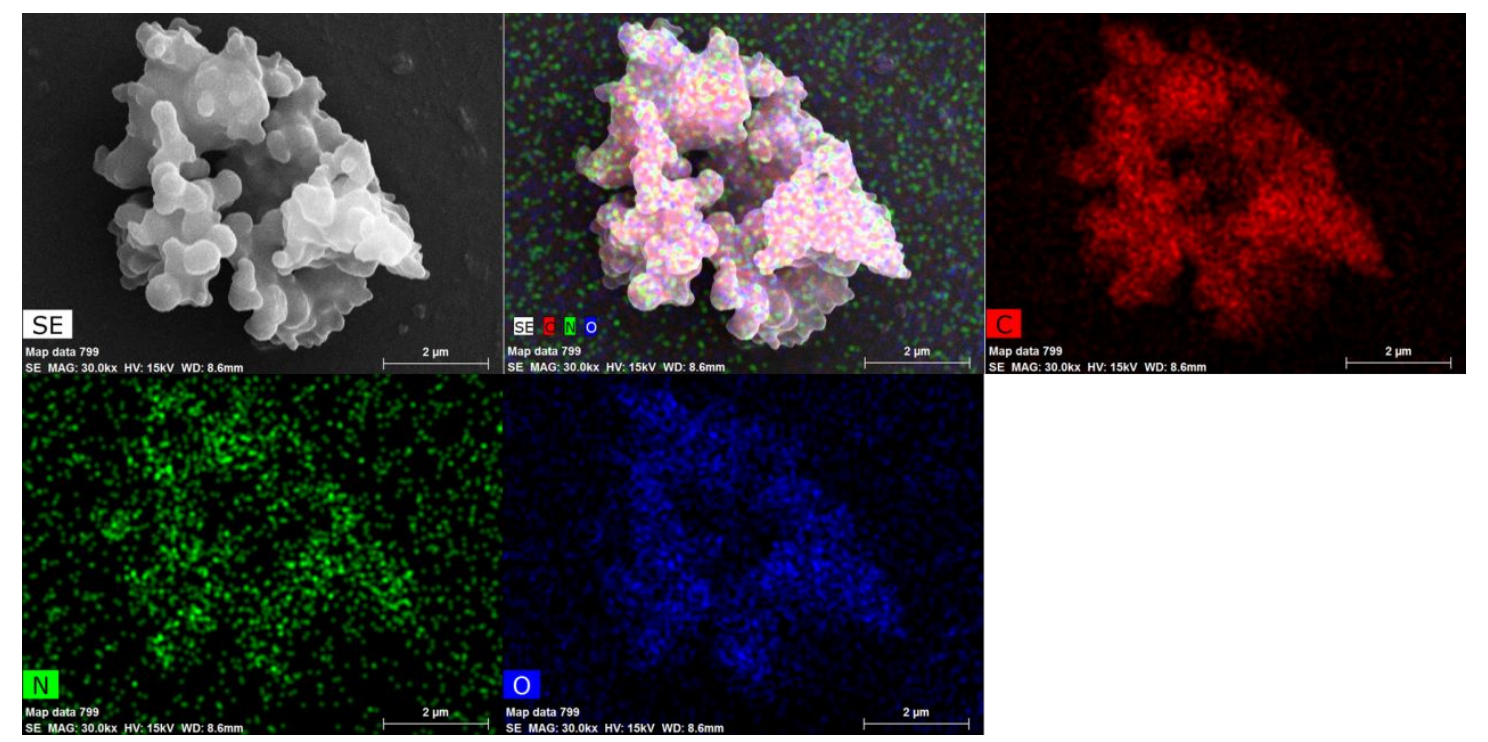

Figure S6. The EDX elemental mapping of TFP-DP-COF. 

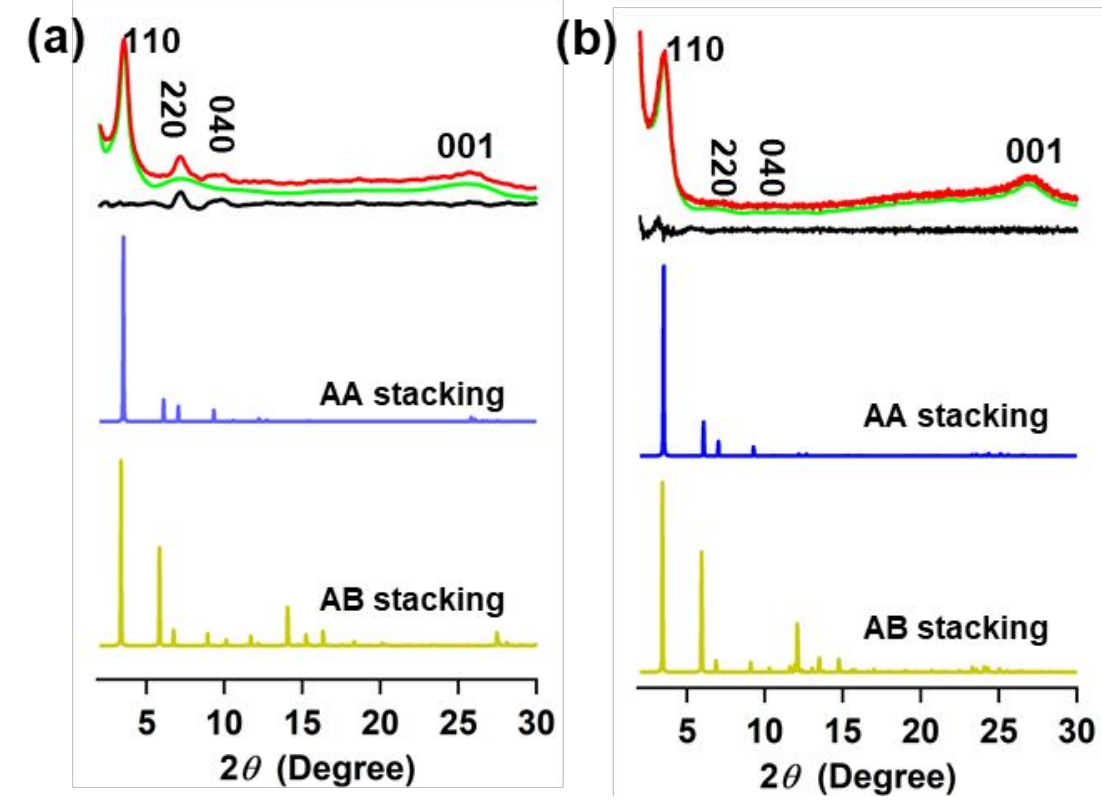

Figure S7. PXRD patterns of TFP-DP-COF (a) and TFP-DB-COF (b) of experimentally observed (red), Pawley refined (green), their difference (black), simulated curves for AA stacking mode (blue), and staggered AB-stacking mode (yellow). 


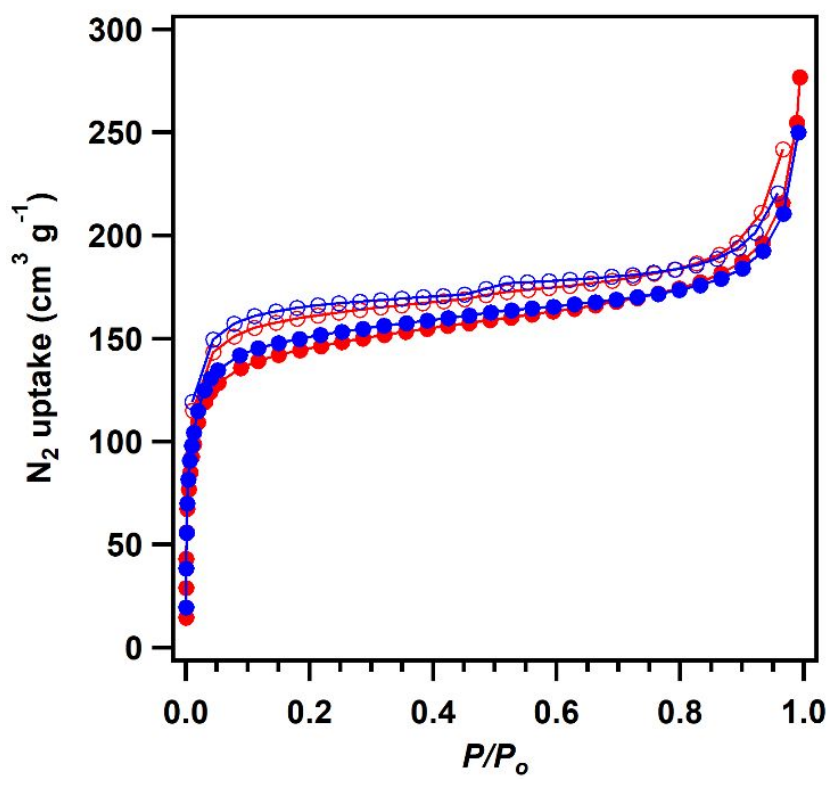

Figure S8. Nitrogen sorption curves of TFP-DP-COF (blue) and TFP-DB-COF (red) at $77 \mathrm{~K}$ (filled circles for adsorption, open circles for desorption).
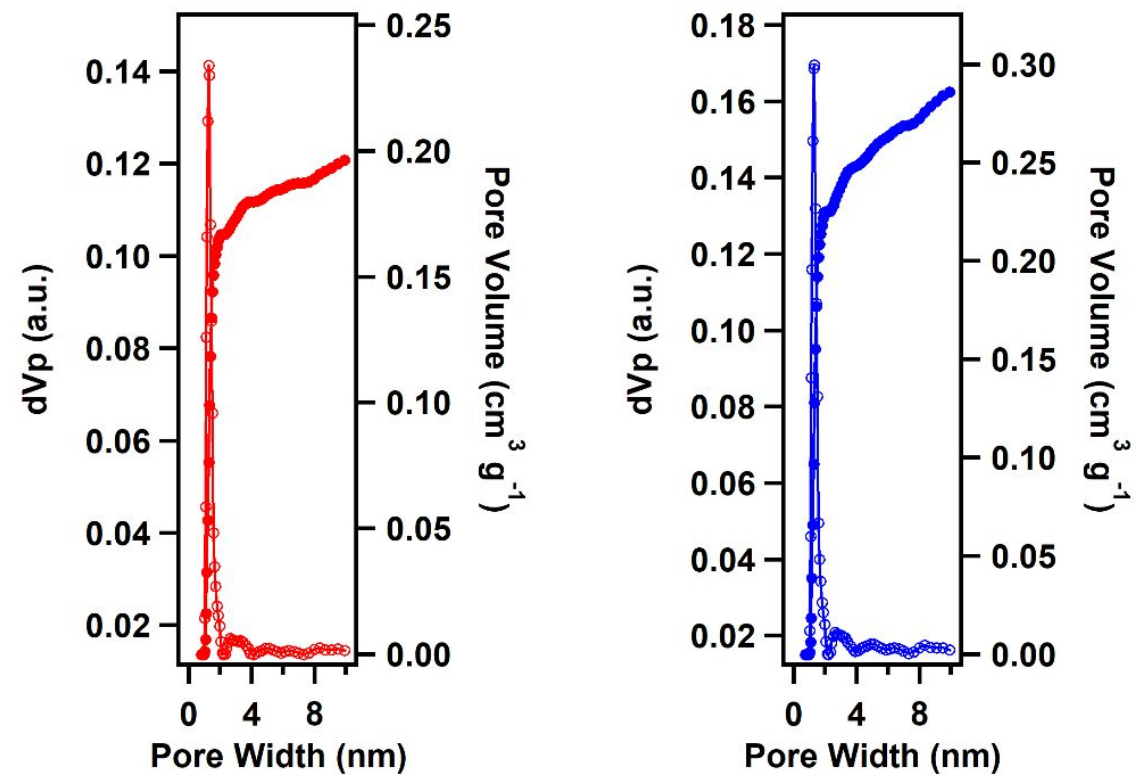

Figure S9. The pore size distribution and pore volume of TFP-DP-COF (blue) and TFP-DB-COF (red). 


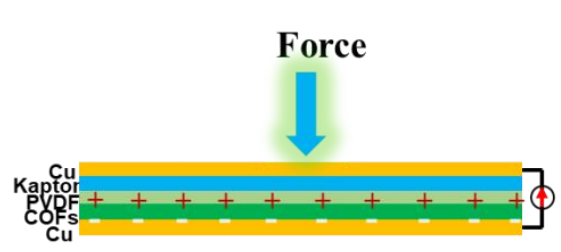

(i) Contacted

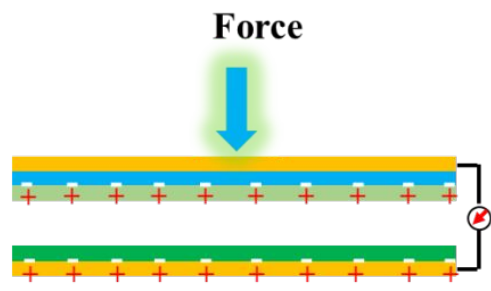

(iv) Compressing

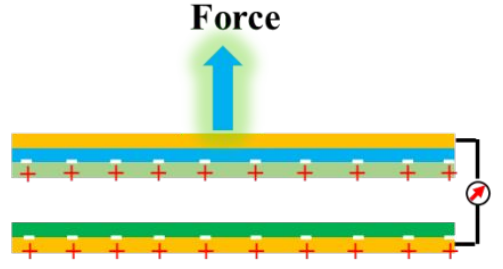

(ii) Separating

Force

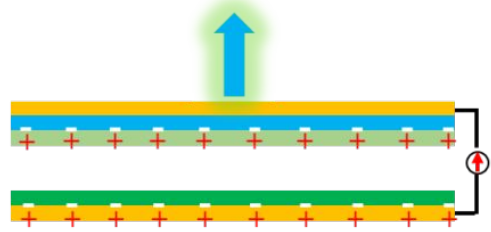

(iii) Separated

Critical parameters for TENG:

$>$ Working frequency

Contact interface

$>$ Environmental factors
Polarity of materials

Charge retention ability of materials

Porosity of materials

Figure S10. Schematic for the working mechanism of vertical contact-separation mode. 

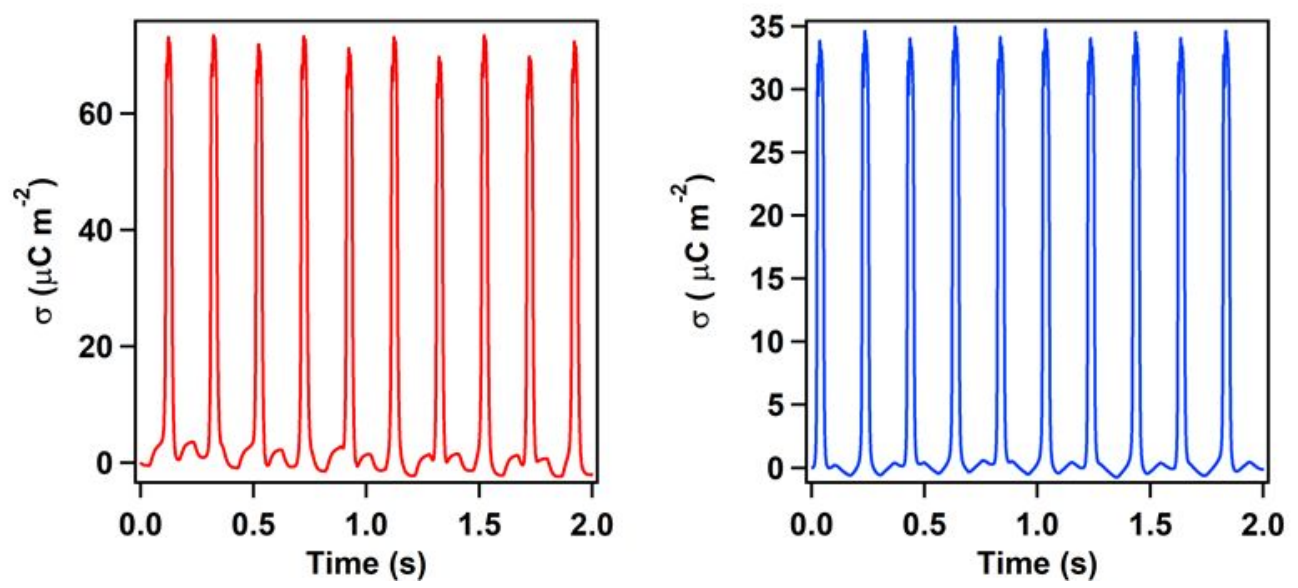

Figure S11. Charge density $(\sigma)$ of TFP-DB-COF (red curve) and TFP-DP-COF (blue curve) based TENG under different external load resistance.

The Charge density $(\sigma)$ can be calculate from the short circuit current using the following equation:

$$
\sigma=\frac{\int i d t}{S}
$$

where $i$ is the short circuit current, $t$ is time and $S$ is the contact area. 
(a)

(b)

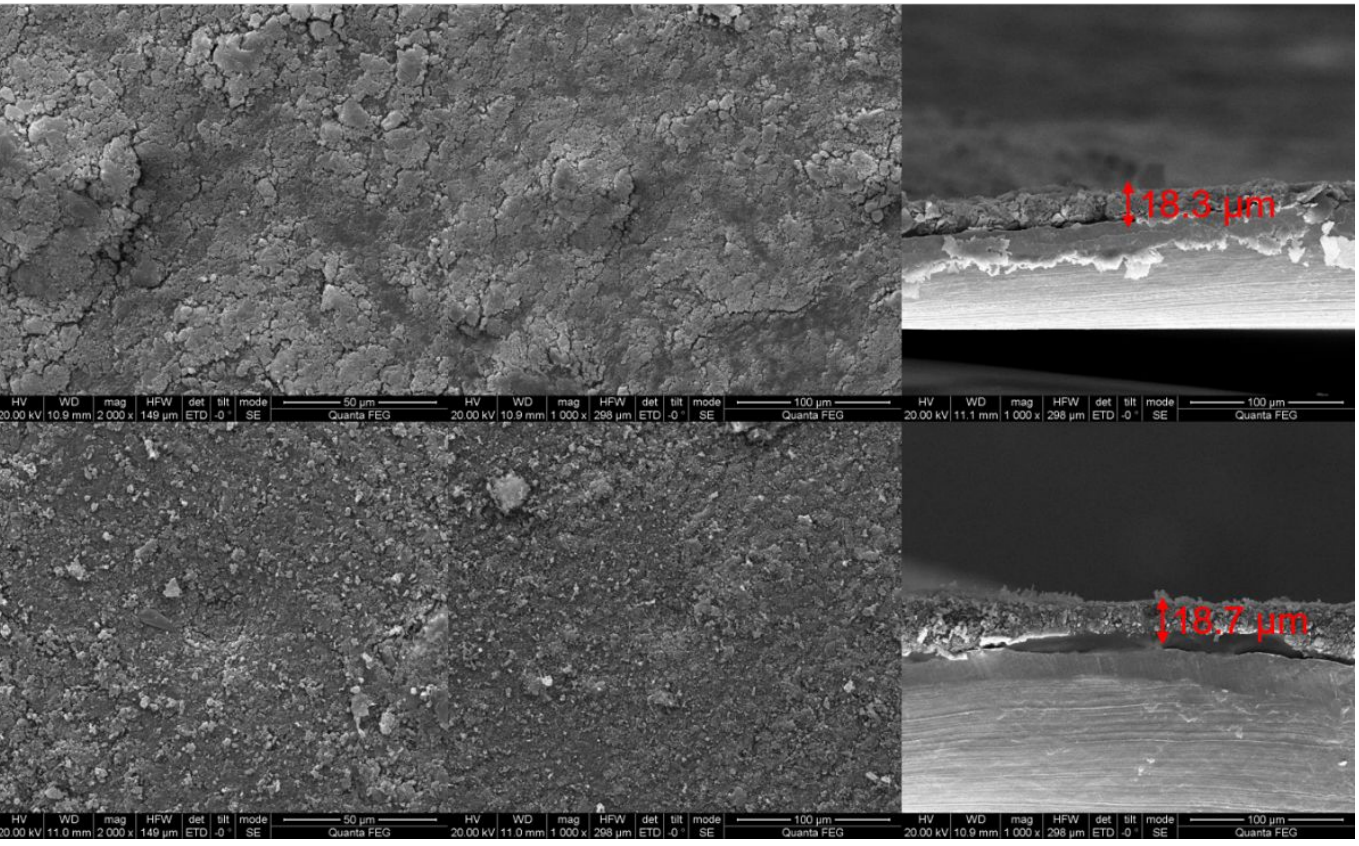

Figure S12. Surface topology and thickness of COF film using Copper $(\mathrm{Cu})$ as substrate: (a) TFP-DB-COF and (b) TFP-DP-COF. 


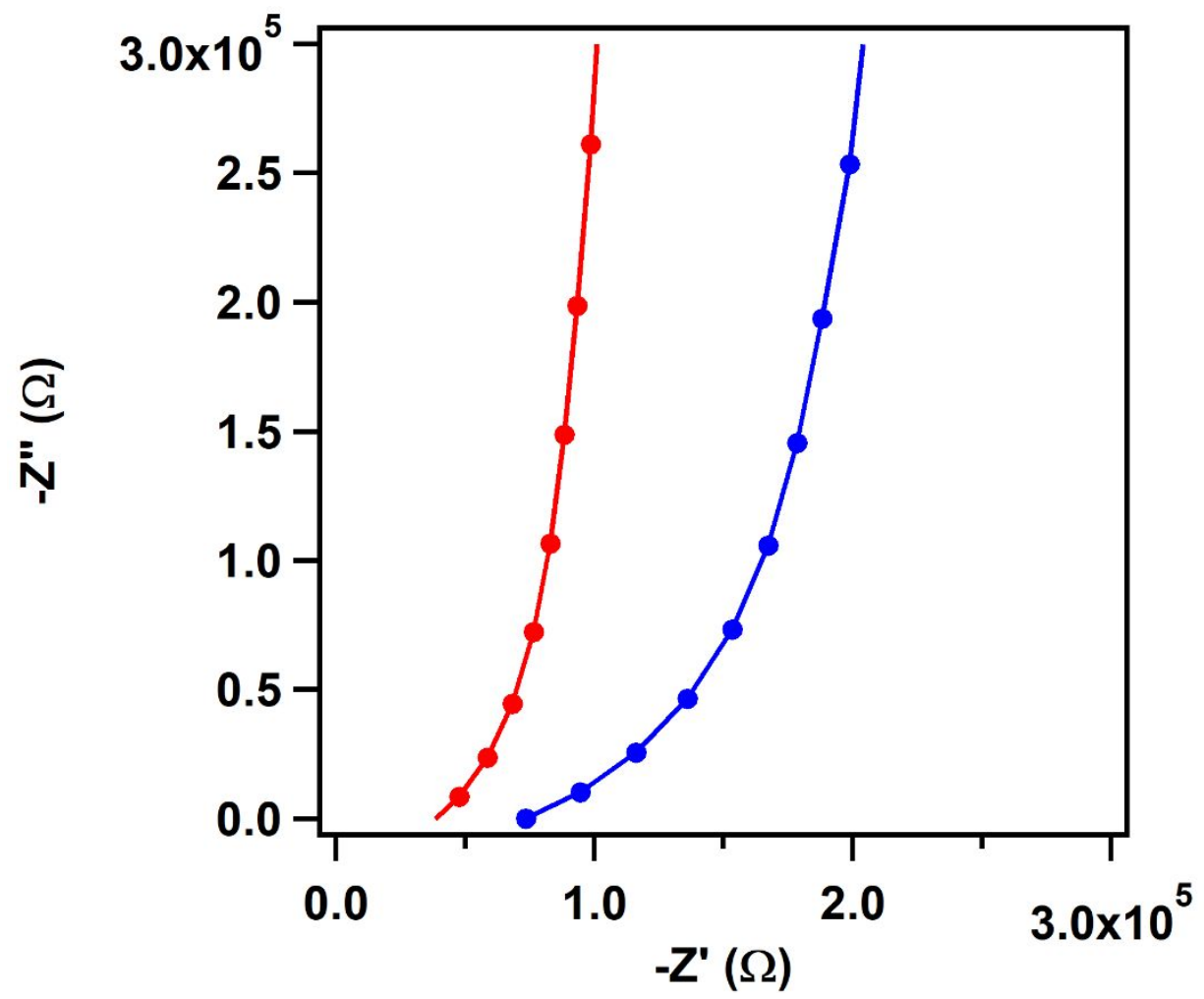

Figure S13. Nyquist plots of TFP-DB-COF (red curve) and TFP-DP-COF (blue curve) under solid state at room temperature.
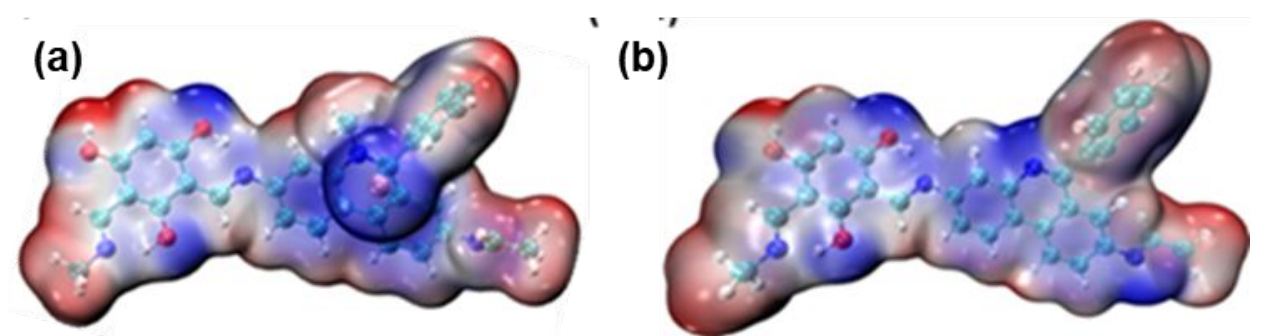

Figure S14. Charge density distribution over the repeat unit of (a) TFP-DB-COF and (b) TFP-DP-COF calculated by Gaussian. 

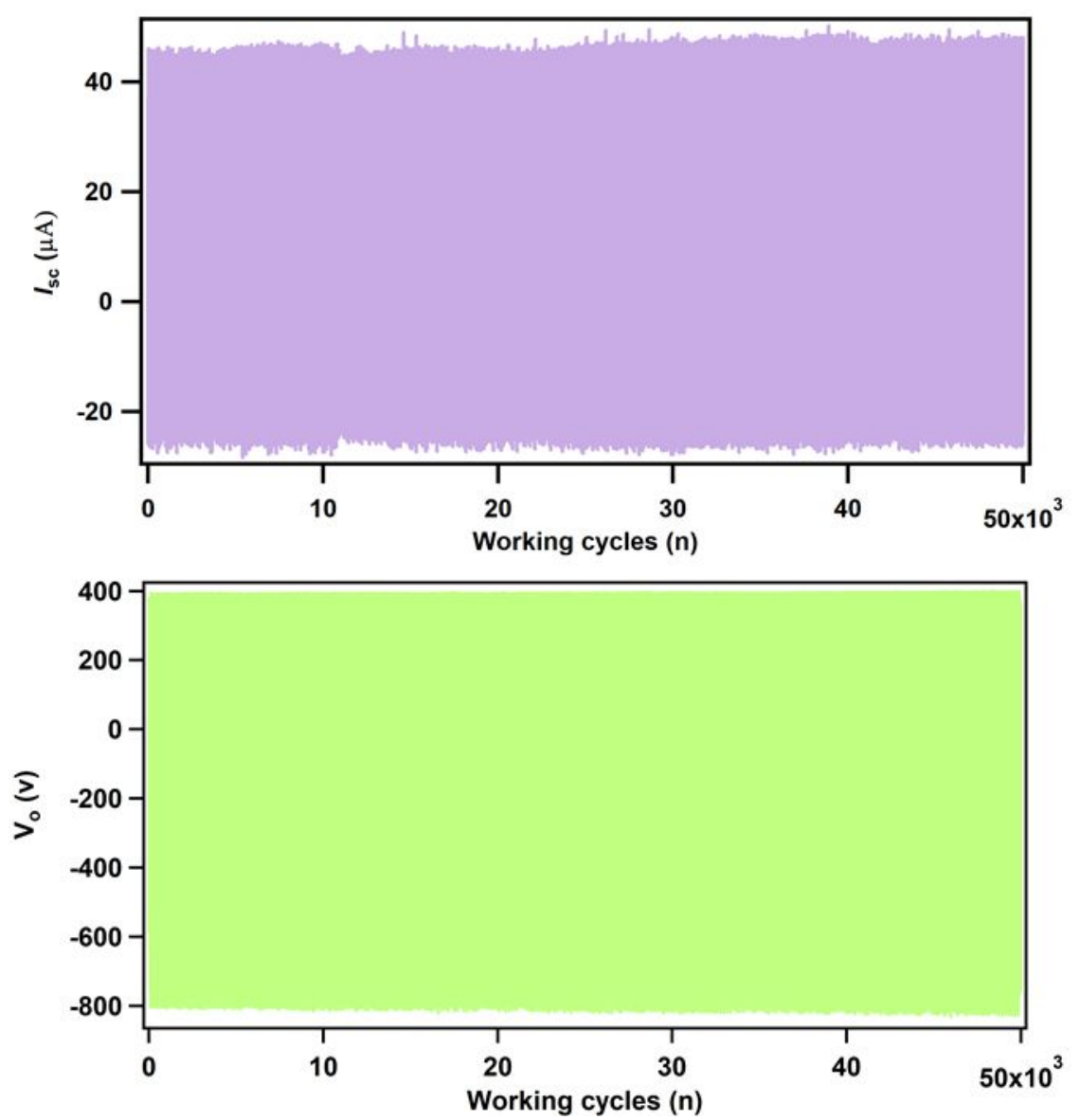

Figure S15. $I_{\mathrm{sc}}$ and $V_{\mathrm{o}}$ at $5 \mathrm{~Hz}$ of TFP-DB-COF based TENG after 50000 working cycles. 


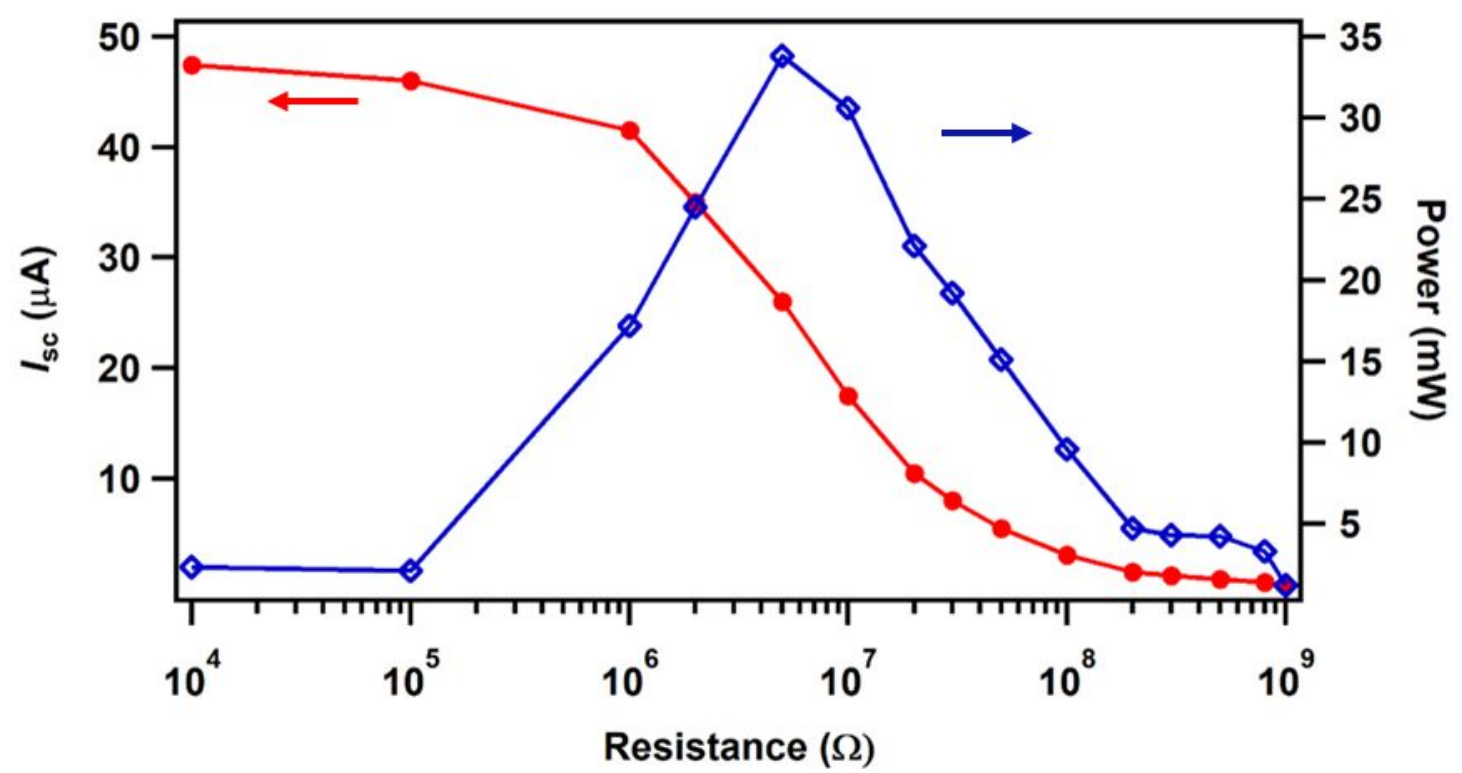

Figure S16. $I_{\mathrm{sc}}$ and $V_{\mathrm{o}}$ of TFP-DB-COF based TENG under different external load resistance. 


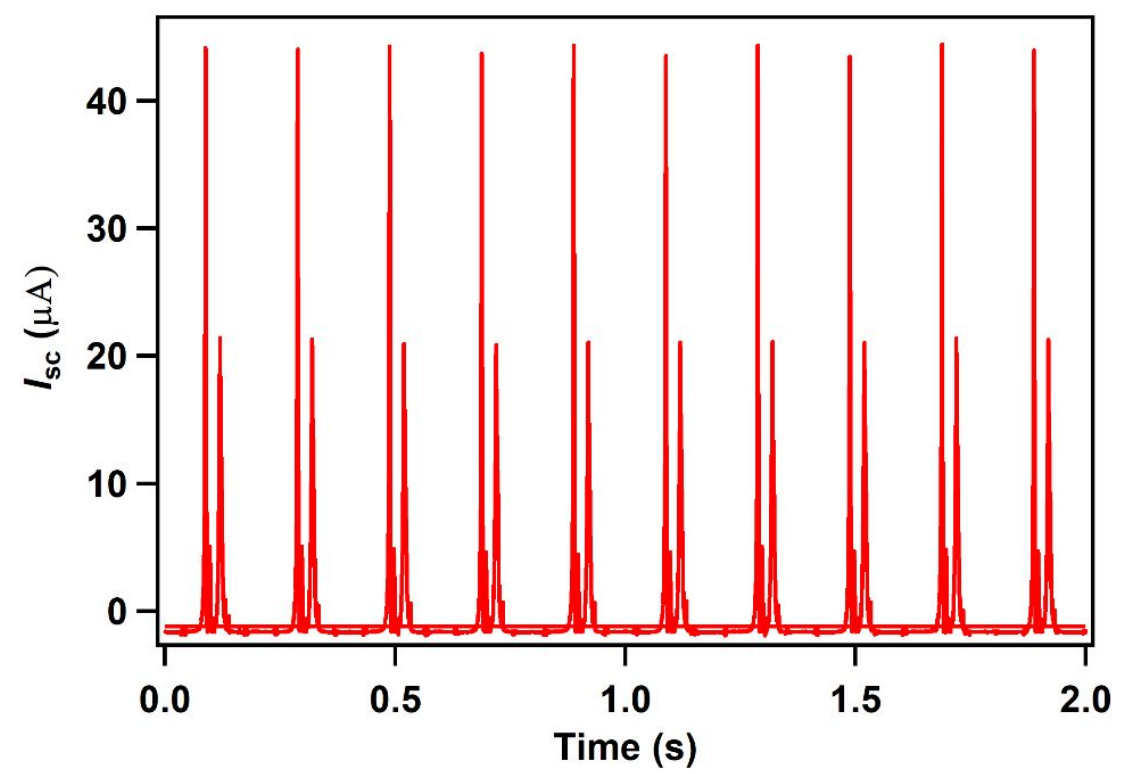

Figure S17. $I_{\mathrm{sc}}$ at $5 \mathrm{~Hz}$ of TFP-DB-COF based TENG with a rectifier bridge to make it in the same direction.

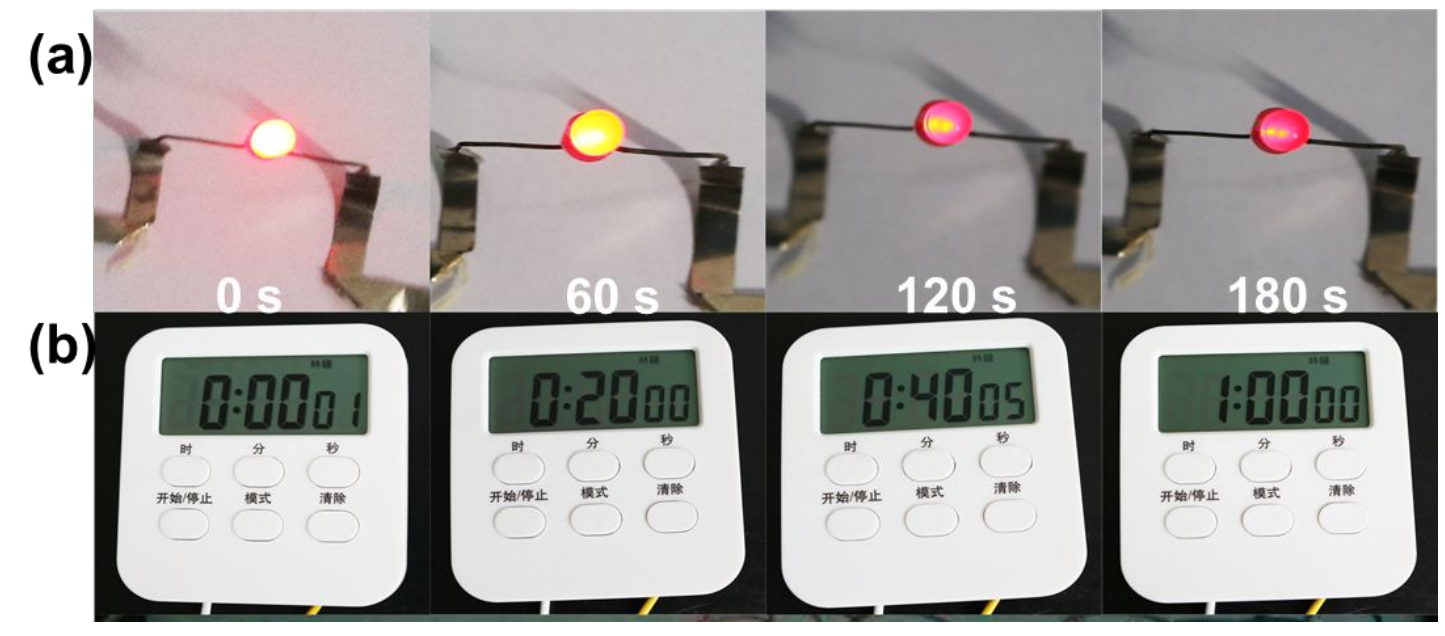

Figure S18. (a) Photographs of the red LED, which is powered by two fully charged commercial capacitors connected in series. (b) The lit-up of the stopwatch for 1 hour with $0.1 \mathrm{~F}$ capacitor. 
Section D. Supporting Tables

Table S1. Elemental analysis

\begin{tabular}{|c|c|c|c|c|}
\hline & & $\mathrm{C}(\%)$ & $\mathrm{H}(\%)$ & $\mathrm{N}(\%)$ \\
\hline \multirow{3}{*}{ TFP-DP-COF } & Calcd. & 76.13 & 3.40 & 9.52 \\
\cline { 2 - 5 } & Found & 75.32 & 3.34 & 9.13 \\
\hline \multirow{3}{*}{ TFP-DB-COF } & Calcd. & 64.89 & 3.36 & 7.83 \\
\cline { 2 - 5 } & Found & 63.79 & 3.12 & 7.65 \\
\hline
\end{tabular}


Table S2. Atomistic coordinates for the AA-stacking mode of TFP-DB-COF optimized using $\mathrm{DFTB}^{+}$method. Space group: P3; $a=29.1006 \AA, b=29.1006 \AA, c=$ $3.8197 \AA$.

\begin{tabular}{|c|c|c|c|c|c|}
\hline N1 & $\mathrm{N}$ & 2.14203 & 1.07812 & 1.30301 & 0 \\
\hline $\mathrm{C} 2$ & $\mathrm{C}$ & 2.19498 & 1.09734 & 1.17585 & 0 \\
\hline $\mathrm{C} 3$ & $\mathrm{C}$ & 2.22087 & 1.06769 & 1.18997 & 0 \\
\hline $\mathrm{C} 4$ & $\mathrm{C}$ & 2.27025 & 1.08535 & 1.02495 & 0 \\
\hline $\mathrm{C} 5$ & $\mathrm{C}$ & 2.29865 & 1.13854 & 0.90429 & 0 \\
\hline C6 & $\mathrm{C}$ & 2.27234 & 1.16843 & 0.89479 & 0 \\
\hline $\mathrm{C} 7$ & $\mathrm{C}$ & 2.22063 & 1.14709 & 1.01991 & 0 \\
\hline $\mathrm{C} 8$ & C & 2.29417 & 1.05296 & 1.00346 & 0 \\
\hline C9 & $\mathrm{C}$ & 2.05466 & 1.01676 & 1.52648 & 0 \\
\hline $\mathrm{C} 10$ & $\mathrm{C}$ & 2.17412 & 0.91309 & 0.7887 & 0 \\
\hline C11 & $\mathrm{C}$ & 2.03775 & 1.05446 & 1.54022 & 0 \\
\hline $\mathrm{C} 12$ & $\mathrm{C}$ & 2.11089 & 1.03306 & 1.45482 & 0 \\
\hline $\mathrm{C} 13$ & $\mathrm{C}$ & 2.26035 & 0.99254 & 0.92906 & 0 \\
\hline C14 & $\mathrm{C}$ & 2.28302 & 0.95948 & 0.88225 & 0 \\
\hline C15 & C & 2.25214 & 0.90514 & 0.81334 & 0 \\
\hline C16 & C & 2.19762 & 0.88179 & 0.772 & 0 \\
\hline C17 & C & 2.20485 & 0.96738 & 0.85805 & 0 \\
\hline O18 & $\mathrm{O}$ & 2.07557 & 1.10848 & 1.56262 & 0 \\
\hline C19 & $\mathrm{C}$ & 2.35295 & 1.16074 & 0.81866 & 0 \\
\hline
\end{tabular}




\begin{tabular}{|c|c|c|c|c|c|}
\hline $\mathrm{C} 20$ & C & 2.38367 & 1.21264 & 0.67989 & 0 \\
\hline $\mathrm{C} 21$ & C & 2.37854 & 1.13153 & 0.9058 & 0 \\
\hline $\mathrm{C} 22$ & C & 2.4346 & 1.1574 & 0.88061 & 0 \\
\hline $\mathrm{C} 23$ & C & 2.46442 & 1.20987 & 0.77168 & 0 \\
\hline $\mathrm{C} 24$ & C & 2.43876 & 1.2369 & 0.66042 & 0 \\
\hline $\mathrm{N} 25$ & $\mathrm{~N}$ & 2.52109 & 1.23489 & 0.77895 & 0 \\
\hline C26 & C & 2.55192 & 1.28333 & 0.88455 & 0 \\
\hline $\mathrm{C} 27$ & C & 2.61061 & 1.30781 & 0.89423 & 0 \\
\hline C28 & C & 2.63647 & 1.2775 & 0.90363 & 0 \\
\hline N29 & $\mathrm{N}$ & 2.34921 & 1.07927 & 1.01632 & 0 \\
\hline $\mathrm{O} 30$ & $\mathrm{O}$ & 2.60834 & 1.22219 & 0.94266 & 0 \\
\hline C31 & C & 2.37689 & 1.05568 & 1.20387 & 0 \\
\hline H32 & $\mathrm{H}$ & 2.20329 & 1.03106 & 1.33126 & 0 \\
\hline H33 & $\mathrm{H}$ & 2.29084 & 1.20858 & 0.79882 & 0 \\
\hline H34 & $\mathrm{H}$ & 2.20026 & 1.16964 & 1.00414 & 0 \\
\hline H35 & $\mathrm{H}$ & 2.13194 & 0.89552 & 0.73963 & 0 \\
\hline H36 & $\mathrm{H}$ & 2.12369 & 1.00455 & 1.49329 & 0 \\
\hline H37 & $\mathrm{H}$ & 2.32473 & 0.9741 & 0.88038 & 0 \\
\hline H38 & $\mathrm{H}$ & 2.27092 & 0.88128 & 0.78345 & 0 \\
\hline H39 & $\mathrm{H}$ & 2.17386 & 0.83984 & 0.7144 & 0 \\
\hline $\mathrm{H} 40$ & $\mathrm{H}$ & 2.1836 & 0.98848 & 0.83897 & 0 \\
\hline H41 & $\mathrm{H}$ & 2.06734 & 1.12698 & 1.75752 & 0 \\
\hline
\end{tabular}




\begin{tabular}{|c|c|c|c|c|c|}
\hline $\mathrm{H} 42$ & $\mathrm{H}$ & 2.36618 & 1.23533 & 0.5851 & 0 \\
\hline $\mathrm{H} 43$ & $\mathrm{H}$ & 2.45628 & 1.13749 & 0.94706 & 0 \\
\hline $\mathrm{H} 44$ & $\mathrm{H}$ & 2.46161 & 1.27681 & 0.55768 & 0 \\
\hline $\mathrm{H} 45$ & $\mathrm{H}$ & 2.53467 & 1.30699 & 0.97 & 0 \\
\hline $\mathrm{H} 46$ & $\mathrm{H}$ & 2.57496 & 1.20871 & 1.08972 & 0 \\
\hline $\mathrm{H} 47$ & $\mathrm{H}$ & 2.39955 & 1.04501 & 1.01899 & 0 \\
\hline $\mathrm{H} 48$ & $\mathrm{H}$ & 2.34928 & 1.02132 & 1.36532 & 0 \\
\hline $\mathrm{H} 49$ & $\mathrm{H}$ & 2.40547 & 1.08396 & 1.39575 & 0 \\
\hline
\end{tabular}


Table S3. Atomistic coordinates for the AB-stacking mode of TFP-DB-COF optimized using $\mathrm{DFTB}^{+}$method. Space group: P1; $a=29.1006 \AA, b=29.1006 \AA, c=$ $7.86 \AA$.

\begin{tabular}{|c|c|c|c|c|c|}
\hline N1 & $\mathrm{N}$ & 0.14203 & 0.07812 & 0.29296 & 0 \\
\hline $\mathrm{C} 2$ & $\mathrm{C}$ & 0.19498 & 0.09734 & 0.25871 & 0 \\
\hline $\mathrm{C} 3$ & $\mathrm{C}$ & 0.22087 & 0.06769 & 0.26251 & 0 \\
\hline $\mathrm{C} 4$ & $\mathrm{C}$ & 0.27025 & 0.08535 & 0.21806 & 0 \\
\hline $\mathrm{C} 5$ & $\mathrm{C}$ & 0.29865 & 0.13854 & 0.18556 & 0 \\
\hline C6 & $\mathrm{C}$ & 0.27234 & 0.16843 & 0.183 & 0 \\
\hline $\mathrm{C} 7$ & $\mathrm{C}$ & 0.22063 & 0.14709 & 0.21671 & 0 \\
\hline $\mathrm{C} 8$ & $\mathrm{C}$ & 0.29417 & 0.05296 & 0.21227 & 0 \\
\hline C9 & $\mathrm{C}$ & 0.05466 & 0.01676 & 0.35315 & 0 \\
\hline $\mathrm{C} 10$ & $\mathrm{C}$ & 0.17412 & 0.91309 & 0.15443 & 0 \\
\hline $\mathrm{C} 11$ & $\mathrm{C}$ & 0.03775 & 0.05446 & 0.35685 & 0 \\
\hline $\mathrm{C} 12$ & $\mathrm{C}$ & 0.11089 & 0.03306 & 0.33385 & 0 \\
\hline $\mathrm{C} 13$ & $\mathrm{C}$ & 0.26035 & 0.99254 & 0.19224 & 0 \\
\hline $\mathrm{C} 14$ & $\mathrm{C}$ & 0.28302 & 0.95948 & 0.17963 & 0 \\
\hline $\mathrm{C} 15$ & $\mathrm{C}$ & 0.25214 & 0.90514 & 0.16107 & 0 \\
\hline $\mathrm{C} 16$ & $\mathrm{C}$ & 0.19762 & 0.88179 & 0.14993 & 0 \\
\hline $\mathrm{C} 17$ & $\mathrm{C}$ & 0.20485 & 0.96738 & 0.17311 & 0 \\
\hline $\mathrm{O} 18$ & $\mathrm{O}$ & 0.07557 & 0.10848 & 0.36288 & 0 \\
\hline C19 & $\mathrm{C}$ & 0.35295 & 0.16074 & 0.1625 & 0 \\
\hline $\mathrm{C} 20$ & $\mathrm{C}$ & 0.38367 & 0.21264 & 0.12512 & 0 \\
\hline $\mathrm{C} 21$ & $\mathrm{C}$ & 0.37854 & 0.13153 & 0.18597 & 0 \\
\hline $\mathrm{C} 22$ & $\mathrm{C}$ & 0.4346 & 0.1574 & 0.17919 & 0 \\
\hline $\mathrm{C} 23$ & $\mathrm{C}$ & 0.46442 & 0.20987 & 0.14985 & 0 \\
\hline $\mathrm{C} 24$ & $\mathrm{C}$ & 0.43876 & 0.2369 & 0.11988 & 0 \\
\hline N25 & $\mathrm{N}$ & 0.52109 & 0.23489 & 0.1518 & 0 \\
\hline $\mathrm{C} 26$ & $\mathrm{C}$ & 0.55192 & 0.28333 & 0.18025 & 0 \\
\hline
\end{tabular}




\begin{tabular}{|c|c|c|c|c|c|}
\hline $\mathrm{C} 27$ & $\mathrm{C}$ & 0.61061 & 0.30781 & 0.18285 & 0 \\
\hline $\mathrm{C} 28$ & $\mathrm{C}$ & 0.63647 & 0.2775 & 0.18539 & 0 \\
\hline N29 & $\mathrm{N}$ & 0.34921 & 0.07927 & 0.21574 & 0 \\
\hline $\mathrm{O} 30$ & $\mathrm{O}$ & 0.60834 & 0.22219 & 0.1959 & 0 \\
\hline C31 & $\mathrm{C}$ & 0.37689 & 0.05568 & 0.26626 & 0 \\
\hline H32 & $\mathrm{H}$ & 0.20329 & 0.03106 & 0.30057 & 0 \\
\hline H33 & $\mathrm{H}$ & 0.29084 & 0.20858 & 0.15716 & 0 \\
\hline H34 & $\mathrm{H}$ & 0.20026 & 0.16964 & 0.21246 & 0 \\
\hline H35 & $\mathrm{H}$ & 0.13194 & 0.89552 & 0.14121 & 0 \\
\hline H36 & $\mathrm{H}$ & 0.12369 & 0.00455 & 0.34421 & 0 \\
\hline H37 & $\mathrm{H}$ & 0.32473 & 0.9741 & 0.17912 & 0 \\
\hline H38 & $\mathrm{H}$ & 0.27092 & 0.88128 & 0.15302 & 0 \\
\hline H39 & $\mathrm{H}$ & 0.17386 & 0.83984 & 0.13442 & 0 \\
\hline $\mathrm{H} 40$ & $\mathrm{H}$ & 0.1836 & 0.98848 & 0.16797 & 0 \\
\hline H41 & $\mathrm{H}$ & 0.06734 & 0.12698 & 0.41538 & 0 \\
\hline $\mathrm{H} 42$ & $\mathrm{H}$ & 0.36618 & 0.23533 & 0.09959 & 0 \\
\hline H43 & $\mathrm{H}$ & 0.45628 & 0.13749 & 0.19708 & 0 \\
\hline $\mathrm{H} 44$ & $\mathrm{H}$ & 0.46161 & 0.27681 & 0.0922 & 0 \\
\hline $\mathrm{H} 45$ & $\mathrm{H}$ & 0.53467 & 0.30699 & 0.20326 & 0 \\
\hline H46 & $\mathrm{H}$ & 0.57496 & 0.20871 & 0.23551 & 0 \\
\hline H47 & $\mathrm{H}$ & 0.39955 & 0.04501 & 0.21646 & 0 \\
\hline H48 & $\mathrm{H}$ & 0.34928 & 0.02132 & 0.30974 & 0 \\
\hline H49 & $\mathrm{H}$ & 0.40547 & 0.08396 & 0.31794 & 0 \\
\hline
\end{tabular}


Table S4. Atomistic coordinates for the AA-stacking mode of TFP-DB-COF optimized using DFTB+ method. Space group: $P 6 ; a=28.9789 \AA, b=28.9789 \AA$, and $c=3.4496 \AA$.

\begin{tabular}{|c|c|c|c|c|c|}
\hline N1 & $\mathrm{N}$ & 0.81041 & 0.41615 & 0 & 0 \\
\hline $\mathrm{C} 2$ & $\mathrm{C}$ & 0.86508 & 0.43949 & 0 & 0 \\
\hline $\mathrm{C} 3$ & $\mathrm{C}$ & 0.89091 & 0.41183 & 0 & 0 \\
\hline $\mathrm{C} 4$ & $\mathrm{C}$ & 0.94477 & 0.43557 & 0 & 0 \\
\hline $\mathrm{C} 5$ & $\mathrm{C}$ & 0.97281 & 0.49064 & 0 & 0 \\
\hline C6 & $\mathrm{C}$ & 0.94567 & 0.51748 & 0 & 0 \\
\hline $\mathrm{C} 7$ & $\mathrm{C}$ & 0.89291 & 0.49206 & 0 & 0 \\
\hline $\mathrm{C} 8$ & $\mathrm{C}$ & 0.02704 & 0.51748 & 0 & 0 \\
\hline C9 & $\mathrm{C}$ & 0.05787 & 0.57142 & 0 & 0 \\
\hline $\mathrm{C} 10$ & $\mathrm{C}$ & 0.05183 & 0.48895 & 0 & 0 \\
\hline C11 & $\mathrm{C}$ & 0.10491 & 0.51263 & 0 & 0 \\
\hline $\mathrm{C} 12$ & $\mathrm{C}$ & 0.13502 & 0.56548 & 0 & 0 \\
\hline C13 & $\mathrm{C}$ & 0.11107 & 0.59496 & 0 & 0 \\
\hline N14 & $\mathrm{N}$ & 0.18938 & 0.5869 & 0 & 0 \\
\hline $\mathrm{C} 15$ & $\mathrm{C}$ & 0.22454 & 0.63463 & 0 & 0 \\
\hline $\mathrm{C} 16$ & $\mathrm{C}$ & 0.28043 & 0.65117 & 0 & 0 \\
\hline $\mathrm{C} 17$ & $\mathrm{C}$ & 0.29622 & 0.61424 & 0 & 0 \\
\hline C18 & $\mathrm{C}$ & 0.97299 & 0.40844 & 0 & 0 \\
\hline N19 & $\mathrm{N}$ & 0.02468 & 0.43774 & 0 & 0 \\
\hline $\mathrm{C} 20$ & $\mathrm{C}$ & 0.61393 & 0.31668 & 0 & 0 \\
\hline $\mathrm{C} 21$ & $\mathrm{C}$ & 0.63032 & 0.28013 & 0 & 0 \\
\hline $\mathrm{C} 22$ & $\mathrm{C}$ & 0.63191 & 0.40798 & 0 & 0 \\
\hline $\mathrm{C} 23$ & $\mathrm{C}$ & 0.64914 & 0.60392 & 0 & 0 \\
\hline $\mathrm{C} 24$ & $\mathrm{C}$ & 0.6628 & 0.65639 & 0 & 0 \\
\hline $\mathrm{C} 25$ & $\mathrm{C}$ & 0.71337 & 0.69601 & 0 & 0 \\
\hline C26 & $\mathrm{C}$ & 0.75279 & 0.68531 & 0 & 0 \\
\hline
\end{tabular}




\begin{tabular}{|l|l|l|l|l|l|}
\hline C27 & C & 0.6911 & 0.59496 & 0 & 0 \\
\hline C28 & C & 0.365 & 0.10666 & 0 & 0 \\
\hline H29 & H & 0.86787 & 0.37273 & 0 & 0 \\
\hline H30 & H & 0.96396 & 0.55836 & 0 & 0 \\
\hline H31 & H & 0.87297 & 0.51339 & 0 & 0 \\
\hline H32 & H & 0.04243 & 0.59659 & 0 & 0 \\
\hline H33 & H & 0.12307 & 0.48965 & 0 & 0 \\
\hline H34 & H & 0.13259 & 0.63602 & 0 & 0 \\
\hline H35 & H & 0.21238 & 0.66211 & 0 & 0 \\
\hline O36 & O & 0.25782 & 0.56283 & 0 & 0 \\
\hline O37 & O & 0.56213 & 0.30232 & 0 & 0 \\
\hline H38 & H & 0.65882 & 0.44768 & 0 & 0 \\
\hline H39 & H & 0.63433 & 0.66793 & 0 & 0 \\
\hline H40 & H & 0.72186 & 0.73522 & 0 & 0 \\
\hline H41 & H & 0.79201 & 0.71601 & 0 & 0 \\
\hline H42 & H & 0.68922 & 0.55931 & 0 & 0 \\
\hline H43 & H & 0.37318 & 0.1458 & 0 & 0 \\
\hline H44 & H & 0.7341 & 0.26824 & 0 & 0 \\
\hline H45 & H & 0.46566 & 0.7312 & 0 & 0 \\
\hline
\end{tabular}


Table S5. Atomistic coordinates for the AB-stacking mode of TFP-DP-COF optimized using DFTB ${ }^{+}$method. Space group: P6; $a=30.2812 \AA, b=30.2812 \AA$, and $c=6.4903 \AA$.

\begin{tabular}{|c|c|c|c|c|c|}
\hline N1 & $\mathrm{N}$ & 0.47706 & 0.7452 & 0 & 0 \\
\hline $\mathrm{C} 2$ & $\mathrm{C}$ & 0.53148 & 0.76762 & 0 & 0 \\
\hline $\mathrm{C} 3$ & $\mathrm{C}$ & 0.55685 & 0.73944 & 0 & 0 \\
\hline $\mathrm{C} 4$ & $\mathrm{C}$ & 0.61083 & 0.76293 & 0 & 0 \\
\hline $\mathrm{C} 5$ & $\mathrm{C}$ & 0.63904 & 0.81807 & 0 & 0 \\
\hline C6 & $\mathrm{C}$ & 0.61214 & 0.8452 & 0 & 0 \\
\hline $\mathrm{C} 7$ & $\mathrm{C}$ & 0.55949 & 0.82011 & 0 & 0 \\
\hline $\mathrm{C} 8$ & $\mathrm{C}$ & -0.30676 & 0.84483 & 0 & 0 \\
\hline C9 & $\mathrm{C}$ & -0.27611 & 0.89879 & 0 & 0 \\
\hline $\mathrm{C} 10$ & $\mathrm{C}$ & -0.28186 & 0.81635 & 0 & 0 \\
\hline C11 & $\mathrm{C}$ & -0.22859 & 0.84038 & 0 & 0 \\
\hline C12 & $\mathrm{C}$ & -0.19865 & 0.89343 & 0 & 0 \\
\hline C13 & $\mathrm{C}$ & -0.223 & 0.92255 & 0 & 0 \\
\hline N14 & $\mathrm{N}$ & -0.14409 & 0.91566 & 0 & 0 \\
\hline $\mathrm{C} 15$ & $\mathrm{C}$ & -0.10978 & 0.96387 & 0 & 0 \\
\hline $\mathrm{C} 16$ & $\mathrm{C}$ & -0.0534 & 0.98249 & 0 & 0 \\
\hline $\mathrm{C} 17$ & $\mathrm{C}$ & -0.03572 & 0.94702 & 0 & 0 \\
\hline C18 & $\mathrm{C}$ & 0.63905 & 0.7357 & 0 & 0 \\
\hline N19 & $\mathrm{N}$ & -0.30909 & 0.765 & 0 & 0 \\
\hline $\mathrm{C} 20$ & $\mathrm{C}$ & 0.28095 & 0.65196 & 0 & 0 \\
\hline $\mathrm{C} 21$ & $\mathrm{C}$ & 0.29541 & 0.6139 & 0 & 0 \\
\hline $\mathrm{C} 22$ & $\mathrm{C}$ & 0.30253 & 0.74436 & 0 & 0 \\
\hline $\mathrm{C} 23$ & $\mathrm{C}$ & 0.32202 & 0.9426 & 0 & 0 \\
\hline $\mathrm{C} 24$ & $\mathrm{C}$ & 0.33599 & 0.99517 & 0 & 0 \\
\hline $\mathrm{C} 25$ & $\mathrm{C}$ & 0.3867 & 1.03463 & 0 & 0 \\
\hline C26 & $\mathrm{C}$ & 0.42598 & 1.02367 & 0 & 0 \\
\hline
\end{tabular}




\begin{tabular}{|c|c|c|c|c|c|}
\hline $\mathrm{C} 27$ & $\mathrm{C}$ & 0.36381 & 0.93335 & 0 & 0 \\
\hline $\mathrm{C} 28$ & $\mathrm{C}$ & 0.02678 & 0.4413 & 0 & 0 \\
\hline H29 & $\mathrm{H}$ & 0.53323 & 0.70026 & 0 & 0 \\
\hline H30 & $\mathrm{H}$ & 0.63046 & 0.88611 & 0 & 0 \\
\hline H31 & $\mathrm{H}$ & 0.53966 & 0.84161 & 0 & 0 \\
\hline H32 & $\mathrm{H}$ & -0.29152 & 0.92401 & 0 & 0 \\
\hline H33 & $\mathrm{H}$ & -0.21019 & 0.81762 & 0 & 0 \\
\hline H34 & $\mathrm{H}$ & -0.20189 & 0.96364 & 0 & 0 \\
\hline H35 & $\mathrm{H}$ & -0.12292 & 0.99053 & 0 & 0 \\
\hline O36 & $\mathrm{O}$ & -0.07296 & 0.89494 & 0 & 0 \\
\hline O37 & $\mathrm{O}$ & 0.22954 & 0.63952 & 0 & 0 \\
\hline H38 & $\mathrm{H}$ & 0.33023 & 0.78391 & 0 & 0 \\
\hline H39 & $\mathrm{H}$ & 0.30763 & 1.0069 & 0 & 0 \\
\hline $\mathrm{H} 40$ & $\mathrm{H}$ & 0.39537 & 1.07395 & 0 & 0 \\
\hline H41 & $\mathrm{H}$ & 0.46534 & 1.05421 & 0 & 0 \\
\hline $\mathrm{H} 42$ & $\mathrm{H}$ & 0.3616 & 0.8975 & 0 & 0 \\
\hline $\mathrm{H} 43$ & $\mathrm{H}$ & 0.03521 & 0.48057 & 0 & 0 \\
\hline H44 & $\mathrm{H}$ & 0.3968 & 0.5978 & 0 & 0 \\
\hline $\mathrm{H} 45$ & $\mathrm{H}$ & 0.13246 & 1.06885 & 0 & 0 \\
\hline N46 & $\mathrm{N}$ & 0.81071 & 0.41221 & 0.5 & 0 \\
\hline $\mathrm{C} 47$ & $\mathrm{C}$ & 0.86529 & 0.43509 & 0.5 & 0 \\
\hline $\mathrm{C} 48$ & $\mathrm{C}$ & 0.89121 & 0.40739 & 0.5 & 0 \\
\hline C49 & $\mathrm{C}$ & 0.94532 & 0.43155 & 0.5 & 0 \\
\hline $\mathrm{C} 50$ & $\mathrm{C}$ & 0.97302 & 0.48673 & 0.5 & 0 \\
\hline C51 & $\mathrm{C}$ & 0.94561 & 0.51328 & 0.5 & 0 \\
\hline C52 & $\mathrm{C}$ & 0.89292 & 0.48763 & 0.5 & 0 \\
\hline C53 & $\mathrm{C}$ & 0.02726 & 0.51411 & 0.5 & 0 \\
\hline C54 & $\mathrm{C}$ & 0.05759 & 0.56815 & 0.5 & 0 \\
\hline C55 & $\mathrm{C}$ & 0.05259 & 0.48611 & 0.5 & 0 \\
\hline
\end{tabular}




\begin{tabular}{|c|c|c|c|c|c|}
\hline C56 & $\mathrm{C}$ & 0.1057 & 0.5103 & 0.5 & 0 \\
\hline C57 & $\mathrm{C}$ & 0.13526 & 0.56322 & 0.5 & 0 \\
\hline C58 & $\mathrm{C}$ & 0.11081 & 0.59222 & 0.5 & 0 \\
\hline N59 & $\mathrm{N}$ & 0.1896 & 0.58522 & 0.5 & 0 \\
\hline C60 & $\mathrm{C}$ & 0.22428 & 0.6332 & 0.5 & 0 \\
\hline C61 & $\mathrm{C}$ & 0.28028 & 0.65051 & 0.5 & 0 \\
\hline C62 & $\mathrm{C}$ & 0.29663 & 0.61399 & 0.5 & 0 \\
\hline C63 & $\mathrm{C}$ & 0.97426 & 0.40504 & 0.5 & 0 \\
\hline N64 & $\mathrm{N}$ & 0.02594 & 0.4349 & 0.5 & 0 \\
\hline C65 & $\mathrm{C}$ & 0.61422 & 0.31848 & 0.5 & 0 \\
\hline C66 & $\mathrm{C}$ & 0.62878 & 0.28044 & 0.5 & 0 \\
\hline C67 & $\mathrm{C}$ & 0.63554 & 0.41106 & 0.5 & 0 \\
\hline C68 & $\mathrm{C}$ & 0.65231 & 0.60932 & 0.5 & 0 \\
\hline C69 & $\mathrm{C}$ & 0.66519 & 0.66163 & 0.5 & 0 \\
\hline $\mathrm{C} 70$ & $\mathrm{C}$ & 0.71552 & 0.70181 & 0.5 & 0 \\
\hline C71 & $\mathrm{C}$ & 0.75546 & 0.69182 & 0.5 & 0 \\
\hline $\mathrm{C} 72$ & $\mathrm{C}$ & 0.69467 & 0.60107 & 0.5 & 0 \\
\hline $\mathrm{C} 73$ & $\mathrm{C}$ & 0.35834 & 0.10338 & 0.5 & 0 \\
\hline H74 & $\mathrm{H}$ & 0.86787 & 0.36806 & 0.5 & 0 \\
\hline H75 & $\mathrm{H}$ & 0.96358 & 0.55418 & 0.5 & 0 \\
\hline H76 & $\mathrm{H}$ & 0.8727 & 0.50875 & 0.5 & 0 \\
\hline H77 & $\mathrm{H}$ & 0.04184 & 0.59308 & 0.5 & 0 \\
\hline H78 & $\mathrm{H}$ & 0.12429 & 0.48769 & 0.5 & 0 \\
\hline H79 & $\mathrm{H}$ & 0.13201 & 0.63336 & 0.5 & 0 \\
\hline H80 & $\mathrm{H}$ & 0.21168 & 0.66032 & 0.5 & 0 \\
\hline O81 & $\mathrm{O}$ & 0.2585 & 0.56226 & 0.5 & 0 \\
\hline O82 & $\mathrm{O}$ & 0.56275 & 0.30585 & 0.5 & 0 \\
\hline H83 & $\mathrm{H}$ & 0.66312 & 0.45073 & 0.5 & 0 \\
\hline H84 & $\mathrm{H}$ & 0.63622 & 0.67256 & 0.5 & 0 \\
\hline
\end{tabular}




\begin{tabular}{|l|l|l|l|l|l|}
\hline H85 & H & 0.72336 & 0.74089 & 0.5 & 0 \\
\hline H86 & H & 0.79454 & 0.72298 & 0.5 & 0 \\
\hline H87 & H & 0.69289 & 0.56541 & 0.5 & 0 \\
\hline H88 & H & 0.36599 & 0.14238 & 0.5 & 0 \\
\hline H89 & H & 0.73049 & 0.26485 & 0.5 & 0 \\
\hline H90 & H & 0.46575 & 0.73281 & 0.5 & 0 \\
\hline
\end{tabular}


Table S6. Electrical conductivity was conducted by a four-probe electrode method with an $\pm 2 \%$ error range.

\begin{tabular}{|c|c|c|c|c|c|c|c|}
\hline Sample & $\begin{array}{c}\text { Height } \\
(\mathrm{mm})\end{array}$ & $\begin{array}{c}\text { Pressure } \\
(\mathrm{MPa})\end{array}$ & $\begin{array}{c}\text { Forward } \\
\text { value } \\
(\Omega \mathrm{cm})\end{array}$ & $\begin{array}{c}\text { Reverse } \\
\text { Value } \\
(\Omega \mathrm{cm})\end{array}$ & $\begin{array}{c}\text { Average } \\
\text { value } \\
(\Omega \mathrm{cm})\end{array}$ & $\begin{array}{c}\text { Temperature } \\
\left({ }^{\circ} \mathrm{C}\right)\end{array}$ & $\begin{array}{c}\text { Humidity } \\
(\% \mathrm{RH})\end{array}$ \\
\hline TFP-DB-COF-1\# & 1.06 & 20 & 47300 & 47300 & 47300 & 25 & 50 \\
\hline TFP-DB-COF-2\# & 1.06 & 20 & 47600 & 47600 & 47600 & 25 & 50 \\
\hline TFP-DB-COF-3\# & 1.06 & 20 & 47400 & 47400 & 47400 & 25 & 50 \\
\hline TFP-DB-COF-4\# & 1.06 & 20 & 47300 & 47300 & 47300 & 25 & 50 \\
\hline TFP-DB-COF-5\# & 1.06 & 20 & 47700 & 47700 & 47700 & 25 & 50 \\
\hline $\begin{array}{c}\text { TFP-DB-COF-averag } \\
\text { e }\end{array}$ & - & - & - & - & 47460 & - & - \\
\hline
\end{tabular}

Four-probe electrode method: The measurement of electrical conductivity was conducted by a four-probe electrode method in which four sharp probes are mechanically pressed on the testing sample surface. The conductivity for TFP-DP-COF was so low that it could not be measured with our equipment. 
Table S7. Comparison of output performance of various materials

\begin{tabular}{|c|c|c|c|}
\hline Materials & $\begin{array}{l}I_{\mathrm{sc}} \\
(\mu \mathrm{A})\end{array}$ & $\begin{array}{l}V_{\mathrm{o}} \\
(\mathrm{V})\end{array}$ & Ref \\
\hline TFP-DB-COF & 47.9 & 815 & \multirow{2}{*}{ This work } \\
\hline TFP-DP-COF & 23.5 & 365 & \\
\hline $\begin{array}{l}\text { Zeolitic imidazole } \\
\text { framework-8 } \\
\text { (ZIF-8) }\end{array}$ & $<10$ & 164 & S8 \\
\hline $\begin{array}{l}\text { Three-layer TENG } \\
(\mathrm{Al} / \mathrm{Au} / \mathrm{Al})\end{array}$ & $<1$ & 80 & S9 \\
\hline $\begin{array}{l}\text { Zeolitic imidazole } \\
\text { framework-8 } \\
\text { (ZIF-8) }\end{array}$ & $<1.1$ & 60 & $\mathrm{~S} 10$ \\
\hline $\begin{array}{l}\text { Tilting-sensitive } \\
\text { triboelectric } \\
\text { nanogenerator } \\
\text { (TS-TENG) }\end{array}$ & 25.97 & 302.87 & $\mathrm{~S} 11$ \\
\hline $\begin{array}{l}\text { All-printed } \\
\text { triboelectric } \\
\text { nanogenerator } \\
\text { (AP-TENG) }\end{array}$ & 13.7 & 98.2 & $\mathrm{~S} 12$ \\
\hline $\begin{array}{c}\text { Nonmetallic } \\
\text { nylon-modified } \\
\text { triboelectric } \\
\text { nanogenerators } \\
\text { (NM-TENG) }\end{array}$ & 138 & 1170 & $\mathrm{~S} 13$ \\
\hline $\begin{array}{c}\text { Cellulose } \\
\text { nanofibrils-based } \\
\text { TENG } \\
\text { (CNF-based TENG) }\end{array}$ & 90 & $30 \mathrm{~V}$ & $\mathrm{~S} 14$ \\
\hline $\begin{array}{c}\mathrm{Ag} \\
\text { nanoparticle-coated }\end{array}$ & 4 & 286 & $\mathrm{~S} 15$ \\
\hline
\end{tabular}




\begin{tabular}{|c|c|c|c|}
\hline $\begin{array}{c}\text { CNF film } \\
\text { (CNF-PEI-Ag) }\end{array}$ & & \\
\hline $\begin{array}{c}\text { Silk-based } \\
\text { triboelectric generator }\end{array}$ & 5.78 & 268 & S16 \\
\hline (TEG) & & & \\
\hline
\end{tabular}




\section{E. Supporting References}

S1. Kresse, G.; Furthmüller, J. Efficiency of Ab-Initio Total Energy Calculations for Metals and Semiconductors Using a Plane-Wave Basis Set. Comput. Mater. Sci. 1996, $6,15-50$.

S2. Kresse, G.; Furthmüller, J. Efficient Iterative Schemes for Ab Initio Total-Energy Calculations Using a Plane-Wave Basis Set. Phys. Rev. B. 1996, 54, 11169-11186.

S3. Perdew, J. P.; Burke, K.; Ernzerhof, M. Generalized Gradient Approximation Made Simple. Phys. Rev. Lett. 1996, 77, 3865-3868.

S4. Kresse, G.; Joubert, D. From Ultrasoft Pseudopotentials to the Projector Augmented-Wave Method. Phys. Rev. B. 1999, 59, 1758-1775.

S5. Blöchl, P. E. Projector Augmented-Wave Method. Phys. Rev. B. 1994, 50, $17953-17979$.

S6. Chong, J. H.; Sauer, M. B.; Patrick, O.; MacLachlan, M. Highly Stable Keto-Enamine Salicylideneanilines. J. Org. Lett. 2003, 5, 3823-3826, Doi:10.1021/o10352714.

S7. Cui, S.; Zheng, Y.; Liang, J.; Wang, D. Conducting polymer PPy nanowire-based triboelectric nanogenerator and its application for self-powered electrochemical cathodic protection. Chem. Sci, 2016, 7, 6477-6483, Doi:10.1039/C6SC02562E.

S8. Khandelwal, G.; Chandrasekhar, A.; Raj, N. P. M. J.; Kim, S. -J. Metal-Organic Framework: A Novel Material for Triboelectric Nanogenerator-Based Self-Powered Sensors and Systems. Adv. Energy. Mater. 2019, 9, 1803581. Doi: 10.1002/aenm.201803581.

S9. Chun, J.; Ye, B. U.; Lee, J. W.; Choi, D.; Kang, C. -Y.; Kim, S. -W.; Wang, Z. L.; Baik, J. M. Boosted output performance of triboelectric nanogenerator via electric double layer effect. Nat. Commun. 2016, 7, 12985. Doi: 10.1038/ncomms12985.

S10. Khandelwal, G.; Raj, N. P. M. J.; Kim, S. -J. Zeolitic Imidazole Framework: Metal-Organic Framework Subfamily Members for Triboelectric Nanogenerators. Adv. Funct. Mater. 2020, 12, 1910162. Doi: 10.1002/adfm.201910162.

S11. Zhong, W.; Xu, L.; Wang, H.; An, J.; Wang, Z. L. Tilting - Sensitive Triboelectric Nanogenerators for Energy Harvesting from Unstable/Fluctuating 
Surfaces. Adv. Funct. Mater. 2019, 45, 1905319. Doi: 10.1002/adfm.201905319.

S12. Seol, M. -L.; Han, J. -W.; Moon, D.; Yoon, K. J.; Hwang, C. S.; Meyyappan, M. All-printed triboelectric nanogenerator. Nano. Energy. 2018, 44, 82-88. Doi: 10.1016/j.nanoen.2017.11.067.

S13. Qian, J.; He, J.; Qian, S.; Zhang, J.; Niu, X.; Fan, X.; Wang, C.; Hou, X.; Mu, J.;

Geng, W.; Chou, X. A Nonmetallic Stretchable Nylon-Modified High Performance Triboelectric Nanogenerator for Energy Harvesting. Adv. Funct. Mater. 2020, 30, 1907414. Doi: 10.1002/adfm.201907414.

S14. Yao, C.; Hernandez, A.; Yu, Y.; Cai, Z.; Wang, X. Triboelectric nanogenerators and power-boards from cellulose nanofibrils and recycled materials. Nano. Energy. 2016, 30, 103-108. Doi: 10.1016/j.nanoen.2016.09.036.

S15. Zhang, C.; Lin, X.; Zhang, N.; Lu, Y.; Wu, Z.; Liu, G.; Nie, S. Chemically functionalized cellulose nanofibrils-based gear-like triboelectric nanogenerator for energy harvesting and sensing. Nano. Energy. 2019, 66, 104126. Doi: 10.1016/j.nanoen.2019.104126.

S16. Zhang, X. -S.; Brugger, J.; Kim, B. A silk-fibroin-based transparent triboelectric generator suitable for autonomous sensor network. Nano. Energy. 2016, 20, 37-47. Doi: 10.1016/j.nanoen.2015.11.036. 